\title{
TWIN POSITIVE SOLUTIONS FOR THREE-POINT BOUNDARY VALUE PROBLEMS OF HIGHER-ORDER DIFFERENTIAL EQUATIONS
}

\author{
YUJI LIU and WEIGAO GE
}

\author{
Received 7 September 2003
}

\begin{abstract}
A new fixed point theorem on cones is applied to obtain the existence of at least two positive solutions of a higher-order three-point boundary value problem for the differential equation subject to a class of boundary value conditions. The associated Green's function is given. Some results obtained recently are generalized.
\end{abstract}

2000 Mathematics Subject Classification: 34B10.

1. Introduction. The multipoint boundary value problems for ordinary differential equations arise in a variety of different areas of applied mathematics and physics. Linear and nonlinear second-order multipoint boundary value problems have been studied by several authors, we refer the reader to $[6,7,8,9,10,11,12,13,14,15]$ and the references therein.

Consider the $n$ th-order two-point boundary value problem

$$
\begin{gathered}
y^{(n)}+f(t, y(t))=0, \quad 0<t<1, \\
y^{(i)}(0)=0, \quad 0 \leq i \leq n-2, \quad y^{(n-1)}(1)=0 .
\end{gathered}
$$

Recently, there have been some papers $[1,2,3,13,14]$ discussing the existence of positive solutions for the BVP (1.1) by using the Guo-Krasnoselskii fixed-point theorem, that is, the expansion/compression-type fixed-point theorem on cones. It was proved that (1.1) has at least one positive solution under certain assumptions ( $f$ is sublinear or suplinear). Agarwal and O'Regan in [3] established the criteria of the existence of two positive solutions of BVP (1.1) when $f_{0}=\lim _{x \rightarrow 0} f(t, x) / x=f_{\infty}=\lim _{x \rightarrow+\infty} f(t, x) / x=+\infty$. However, the problem of existence of multiple positive solutions of BVP (1.1) remains open when either $f_{0}=\lim _{x \rightarrow 0} f(t, x) / x$ or $f_{\infty}=\lim _{x \rightarrow+\infty} f(t, x) / x$ does not exist.

On the other hand, to the best of our knowledge, few authors have studied the existence of multiple positive solutions for higher-order multipoint boundary value problems. It is an interesting problem and one of the future research directions to discuss the solvability of the $n$ th-order differential equations

$$
x^{(n)}(t)=f(x(t)), \quad 0<t<1,
$$

satisfying either $k$-point right focal boundary value conditions or $k$-point boundary value conditions $[4,5]$. 
Motivated by the results $[1,2,3,4,5]$, we, in this paper, study the existence of multiple positive solutions for the $n$ th-order three-point boundary value problems consisting of the differential equation

$$
y^{(n)}(t)+f\left(t, y(t), y^{\prime}(t), \ldots, y^{(n-2)}(t)\right)=0, \quad 0<t<1,
$$

and following boundary value conditions:

$$
y^{(i)}(0)=0, \quad i=0,1, \ldots, n-2, \quad y^{(n-1)}(1)=\alpha y^{(n-1)}(\eta) .
$$

We give the following assumptions:

$\left(\mathrm{H}_{1}\right) f:[0,1] \times R_{+}^{n-1} \rightarrow[0,+\infty)$ is continuous, where $R_{+}=[0,+\infty)$,

$\left(\mathrm{H}_{2}\right) \quad 1>\alpha \geq 0,0<\eta<1$, and $n \geq 2$, but fixed.

We will impose growth conditions on $f$ to obtain two positive solutions of BVP (1.3)(1.4). The main results in $[1,3,13,14]$ are corollaries of our theorems.

This paper is organized as follows. In Section 2, we first introduce some definitions and a fixed-point theorem, which is the generalized form of the Leggett-Williams fixedpoint theorem, founded in Avery and Henderson [6], and then we present our main results. Several corollaries to illustrate the main results are given in Section 3.

2. Main results. For convenience, we first introduce some definitions in Banach spaces, such as in [6, 9], and a fixed theorem, which is a generalization of the LeggettWilliams fixed point theorem, see Avery and Henderson [6]. The main results and their proofs will be presented at the end of this section.

DEFINITION 2.1. Let $X$ be a real Banach space; a nonempty closed convex set $P \subset X$ is called a cone of $X$ if it satisfies the following conditions:

(i) $x \in P, \lambda \geq 0$ implies $\lambda x \in P$,

(ii) $x \in P,-x \in P$ implies $x=0$.

Every cone $P \subset X$ induces an ordering in $X$, which is given by $x \leq y$ if and only if $y-x \in P[6]$.

Definition 2.2. A map $\psi: P \rightarrow[0,+\infty)$ is called a nonnegative, continuous, increasing functional, provided $\psi$ is nonnegative and continuous and satisfies $\psi(x) \leq \psi(y)$ for all $x, y \in P$ with $x \leq y$.

DEFINITION 2.3. An operator is called completely continuous if it is continuous and maps bounded sets into precompact sets. Denote

$$
\begin{aligned}
P(\psi, d) & =\{x \in P: \psi(x)<d\}, \\
\partial P(\psi, d) & =\{x \in P: \psi(x)=d\}, \\
\overline{P(\psi, d)} & =\{x \in P: \psi(x) \leq d\} .
\end{aligned}
$$

LEMMA 2.4 [6]. Let $X$ be a real Banach space, $P$ a cone of $X, \gamma$ and $\phi$ two nonnegative increasing continuous maps, $\theta$ a nonnegative continuous map with $\theta(0)=0$. Suppose 
there are two positive numbers $c$ and $M$ such that

$$
\gamma(x) \leq \theta(x) \leq \phi(x), \quad\|x\| \leq M \gamma(x) \quad \text { for } x \in \overline{P(\gamma, c)} .
$$

Again, assume $T: \overline{P(\gamma, c)} \rightarrow P$ is completely continuous, and that there are positive numbers $0<a<b<c$ such that

$$
\theta(\lambda x) \leq \lambda \theta(x) \quad \forall \lambda \in[0,1], x \in \partial P(\theta, b)
$$

and

(i) $\gamma(T x)>c$ for $x \in \partial P(\gamma, c)$,

(ii) $\theta(T x)<b$ for $x \in \partial P(\theta, b)$,

(iii) $\phi(T x)>a$ and $P(\phi, a) \neq \varnothing$ for $x \in \partial P(\phi, a)$.

Then $T$ has at least two fixed points $x_{1}$ and $x_{2} \in \overline{P(\gamma, c)}$ satisfying

$$
a<\phi\left(x_{1}\right), \quad \theta\left(x_{1}\right)<b, \quad b<\theta\left(x_{2}\right), \quad \gamma\left(x_{2}\right)<c .
$$

The following lemma is similar to Lemma 2.4, whose proof is omitted.

LEMMA 2.5. Let $X$ be a real Banach space, $P$ a cone of $X, \gamma$ and $\phi$ two nonnegative increasing continuous maps, $\theta$ a nonnegative continuous map, and $\theta(0)=0$. Suppose there are two positive numbers $c$ and $M$ such that

$$
\gamma(x) \leq \theta(x) \leq \phi(x), \quad\|x\| \leq M \gamma(x) \quad \text { for } x \in \overline{P(\gamma, c)} .
$$

Again, assume $T: \overline{P(\gamma, c)} \rightarrow P$ is completely continuous, and that there are positive numbers $0<a<b<c$ such that

$$
\theta(\lambda x) \leq \lambda \theta(x) \quad \forall \lambda \in[0,1], x \in \partial P(\theta, b)
$$

and

(i) $\gamma(T x)<c$ for $x \in \partial P(\gamma, c)$,

(ii) $\theta(T x)>b$ for $x \in \partial P(\theta, b)$,

(iii) $\phi(T x)<a$ and $P(\phi, a) \neq \varnothing$ for $x \in \partial P(\phi, a)$.

Then $T$ has at least two fixed points $x_{1}$ and $x_{2} \in \overline{P(\gamma, c)}$ satisfying

$$
a<\phi\left(x_{1}\right), \quad \theta\left(x_{1}\right)<b, \quad b<\theta\left(x_{2}\right), \quad \gamma\left(x_{2}\right)<c .
$$

To be able to apply Lemmas 2.4 and 2.5 , we must define an operator on a cone in a suitable Banach space. In order to do this, we first observe the Green functions for the above $n$ th-order three-point boundary value problem.

LEMmA 2.6. Suppose $N=1-\alpha \neq 0$. If $y \in C[0,1]$, then the problem

$$
\begin{gathered}
u^{(n)}(t)+y(t)=0, \quad 0 \leq t \leq 1, \\
u^{(i)}(0)=0, \quad i=0,1, \ldots, n-2, \quad u^{(n-1)}(1)=\alpha u^{(n-1)}(\eta),
\end{gathered}
$$


has the unique solution

$$
\begin{aligned}
u(t) & =-\int_{0}^{t} \frac{(t-s)^{n-1}}{(n-1) !} y(s) d s+\frac{t^{n-1}}{(n-1) !(1-\alpha)}\left[\int_{0}^{1} y(s) d s-\alpha \int_{0}^{\eta} y(s) d s\right] \\
& =\int_{0}^{1} G(t, s ; \eta) y(s) d s,
\end{aligned}
$$

where $M=(n-1) !(1-\alpha)$ and

$$
G(t, s ; \eta)=\frac{1}{M} \begin{cases}t^{n-1}-(1-\alpha)(t-s)^{n-1}-\alpha t^{n-1}, & 0 \leq s \leq t<\eta<1 \text { or } 0 \leq s \leq \eta \leq t \leq 1, \\ t^{n-1}-\alpha t^{n-1}, & 0 \leq t \leq s \leq \eta<1, \\ t^{n-1}-(1-\alpha)(t-s)^{n-1}, & 0 \leq \eta \leq s \leq t \leq 1, \\ t^{n-1}, & 0<\eta \leq t \leq s \leq 1 \text { or } 0 \leq t<\eta \leq s \leq 1 .\end{cases}
$$

Furthermore, if $y(t) \geq 0$ for $t \in[0,1]$, then the unique solution $u$ satisfies $u(t) \geq 0$ for $t \in[0,1]$.

Proof. Suppose that

$$
u(t)=-\int_{0}^{t} \frac{(t-s)^{n-1}}{(n-1) !} y(s) d s+A t^{n-1}
$$

is the unique solution of (2.8). One gets

$$
-\int_{0}^{1} y(s) d s+A(n-1) !=-\alpha \int_{0}^{\eta} y(s) d s+\alpha(n-1) ! A
$$

and then

$$
A=\frac{1}{1-\alpha}\left[\int_{0}^{1} \frac{1}{(n-1) !} y(s) d s-\alpha \int_{0}^{\eta} \frac{1}{(n-1) !} y(s) d s\right]
$$

Substitute $A$ into (2.11). Then the first part of the lemma is complete.

To prove that $u(t) \geq 0$ for $t \in[0,1]$, it suffices to prove that $G(t, s ; \eta) \geq 0$ for $(t, s) \in$ $[0,1] \times[0,1]$. This is simple and is omitted.

Let $E$ denote the Banach space $C^{n-2}[0,1]$ with the norm

$$
\|y\|=\max \left\{\|y\|_{\infty}, \ldots,\left\|y^{(n-2)}\right\|_{\infty}\right\} .
$$

We note that, for $y \in E$ with $y^{(i)}(0)=0$ for $i=0,1, \ldots, n-2$,

$$
\begin{gathered}
|y(t)|=|y(t)-y(0)|=\left|t y^{\prime}(\xi)\right| \leq\left|y^{\prime}(\xi)\right| \leq\left\|y^{\prime}\right\|_{\infty}, \\
\left|y^{\prime}(t)\right|=\left|y^{\prime}(t)-y^{\prime}(0)\right|=\left|t y^{\prime \prime}\left(\xi_{1}\right)\right| \leq\left|y^{\prime \prime}\left(\xi_{1}\right)\right| \leq\left\|y^{\prime \prime}\right\|_{\infty} .
\end{gathered}
$$

Hence, $\|y\|_{\infty} \leq\left\|y^{\prime}\right\|_{\infty}$ and $\left\|y^{\prime}\right\|_{\infty} \leq\left\|y^{\prime \prime}\right\|_{\infty}$. By bootstrapping, one sees that

$$
\|y\|_{\infty} \leq\left\|y^{\prime}\right\|_{\infty} \leq \cdots \leq\left\|y^{(p-1)}\right\|_{\infty} \leq \cdots \leq\left\|y^{(n-2)}\right\|_{\infty} .
$$


So

$$
\|y\|=\left\|y^{(n-2)}\right\|_{\infty} .
$$

Define the subset of $E$ by

$$
\begin{aligned}
P= & \left\{y \in E: y^{(i)}(0)=0, i=0,1, \ldots, n-2, y^{(n-2)}(t) \geq t\left\|y^{(n-2)}\right\|_{\infty},\right. \\
& \left.y^{(n-2)}(t) \text { is nondecreasing on }[0,1]\right\} .
\end{aligned}
$$

Define an operator $T$ by

$$
\begin{aligned}
T x(t)=- & \int_{0}^{t} \frac{(t-s)^{n-1}}{(n-1) !} f\left(s, x(s), x^{\prime}(s), \ldots, x^{(n-2)}(s)\right) d s \\
+\frac{t^{n-1}}{(1-\alpha)(n-1) !}[ & \int_{0}^{1} f\left(s, x(s), x^{\prime}(s), \ldots, x^{(n-2)}(s)\right) d s \\
& \left.\quad-\alpha \int_{0}^{\eta} f\left(s, x(s), x^{\prime}(s), \ldots, x^{(n-2)}(s)\right) d s\right]
\end{aligned}
$$

for $x \in E$. Then

$$
\begin{aligned}
(T x)^{(n-2)}(t)=- & \int_{0}^{t}(t-s) f\left(s, x(s), x^{\prime}(s), \ldots, x^{(n-2)}(s)\right) d s \\
+ & \frac{t}{1-\alpha}\left[\int_{0}^{1} f\left(s, x(s), x^{\prime}(s), \ldots, x^{(n-2)}(s)\right) d s\right. \\
& \left.-\alpha \int_{0}^{\eta} f\left(s, x(s), x^{\prime}(s), \ldots, x^{(n-2)}(s)\right) d s\right], \\
(T x)^{(n-1)}(t)=-\int_{0}^{t} f\left(s, x(s), x^{\prime}(s), \ldots, x^{(n-2)}(s)\right) d s & +\frac{1}{1-\alpha}\left[\int_{0}^{1} f\left(s, x(s), x^{\prime}(s), \ldots, x^{(n-2)}(s)\right) d s\right. \\
& \left.-\alpha \int_{0}^{\eta} f\left(s, x(s), x^{\prime}(s), \ldots, x^{(n-2)}(s)\right) d s\right], \\
(T x)^{(n)}(t)=- & f\left(t, x(t), x^{\prime}(t), \ldots, x^{(n-2)}(t)\right) .
\end{aligned}
$$

Hence, we get the following lemma.

LEMmA 2.7. Assume $\left(\mathrm{H}_{1}\right)$ and $\left(\mathrm{H}_{2}\right)$. Then

(i) $P$ is a cone in Banach space $E$;

(ii) $T P \subset P$ and $T$ is completely continuous;

(iii) if $x \in P$, then $T x^{(i)}(0)=0, i=0,1, \ldots, n-2$;

(iv) $T x(t) \geq 0, \ldots,(T x)^{(n-1)}(t) \geq 0,(T x)^{(n)}(t) \leq 0$ for all $t \in(0,1)$;

(v) $y$ is a positive solution of $B V P(1.3)$ and (1.4) if and only if $y$ is a fixed point of the operator $T$ in the $P$.

Proof. The proofs of (i)-(v) are simple and are omitted. 
From now on, fix $l$ such that $0<\eta<l<1$, and define the nonnegative, increasing, continuous functionals $\gamma, \theta$, and $\phi$ by

$$
\begin{aligned}
& \gamma(u)=\min _{\eta \leq t \leq l} u^{(n-2)}(t)=u^{(n-2)}(\eta), \\
& \theta(u)=\max _{0 \leq t \leq \eta} u^{(n-2)}(t)=u^{(n-2)}(\eta), \\
& \phi(u)=\min _{l \leq t \leq 1} u^{(n-2)}(t)=u^{(n-2)}(l)
\end{aligned}
$$

for every $u \in P$. We see that $\gamma(u)=\theta(u) \leq \phi(u)$. In addition, for each $u \in P, \gamma(u)=$ $u^{(n-2)}(\eta) \geq \eta u^{(n-2)}(1)=\eta\left\|u^{(n-2)}\right\|_{\infty}$. Hence,

$$
\|u\|=\left\|u^{(n-2)}\right\|_{\infty} \leq \frac{1}{\eta} \gamma(u) \quad \forall u \in P .
$$

We also find that

$$
\theta(\lambda u)=\lambda \theta(u) \text { for } \lambda \in[0,1], u \in P(\theta, b)
$$

Finally, for notational convenience, we denote

$$
\lambda=\frac{\eta(1-\eta)}{1-\alpha}, \quad \xi=\frac{1}{2} \eta^{2}+\frac{\eta(1-\eta)}{1-\alpha}, \quad \lambda_{l}=\frac{l(1-l)}{1-\alpha} .
$$

We now present our first result of this paper.

THEOREM 2.8. Suppose $0<a<\left(\lambda_{l} / \xi\right) b<\eta\left(\lambda_{l} / \xi\right) c$, and $f$ satisfies the following conditions:

(A) $f\left(t, w_{0}, w_{1}, \ldots, w_{n-2}\right)>c / \lambda$ for $\left(t, w_{0}, w_{1}, \ldots, w_{n-2}\right) \in[\eta, 1] \times R_{+}^{n-2} \times[c, c / \eta]$;

(B) $f\left(t, w_{0}, w_{1}, \ldots, w_{n-2}\right)<b / \xi$ for $\left(t, w_{0}, w_{1}, \ldots, w_{n-2}\right) \in[0,1] \times R_{+}^{n-2} \times[0, b / \eta]$;

(C) $f\left(t, w_{0}, w_{1}, \ldots, w_{n-2}\right)>a / \lambda_{l}$ for $\left(t, w_{0}, w_{1}, \ldots, w_{n-2}\right) \in[l, 1] \times R_{+}^{n-2} \times[a, a / l]$.

Then the BVP (1.3)-(1.4) admits at least two positive solutions $u_{1}, u_{2}$ such that

$$
a<\phi\left(u_{1}\right) \quad \text { with } \theta\left(u_{1}\right)<b, \quad b<\theta\left(u_{2}\right) \quad \text { with } \gamma\left(u_{2}\right)<c \text {. }
$$

Proof. To begin, we define a completely continuous operator $T: P \rightarrow E$ as above for every $u \in P$. Obviously, $w(t)=T u(t) \geq 0$ for $t \in[0,1]$.

From the definition of $T$ and Lemma 2.7, we claim that for each $u \in P, w=T u \in P$ and satisfies (1.4) and $w(1)$ is the maximum value of $w$ on $[0,1]$.

It is well known that each fixed point of $T$ in $P$ is a solution of (1.3)-(1.4). We proceed to verify that the conditions of Lemma 2.4 are met.

As a result of Lemma 2.7, we conclude that $T: \overline{P(\gamma, c)} \rightarrow P$ and $T$ is completely continuous. We now show that (i), (ii), (iii) of Lemma 2.4 are satisfied.

Firstly, we prove that Lemma 2.4(i) is satisfied. For each $u \in \partial P(\gamma, c)$,

$$
\gamma(u)=\min _{\eta \leq t \leq l} u^{(n-2)}(t)=u^{(n-2)}(\eta)=c .
$$


Then $u^{(n-2)}(t) \geq c$ for $\eta \leq t \leq 1$. Recalling that

$$
\begin{gathered}
\|u\|=\left\|u^{(n-2)}\right\|_{\infty} \leq \frac{1}{\eta} \gamma(u)=\frac{1}{\eta} c, \\
u^{(i)}(t) \geq 0 \quad \forall t \in[0,1], i=0,1, \ldots, n-2,
\end{gathered}
$$

we have

$$
c \leq u^{(n-2)}(t) \leq \frac{1}{\eta} c \text { for } \eta \leq t \leq 1 .
$$

As a consequence of (A),

$$
f\left(s, u(s), u^{\prime}(s), \ldots, u^{(n-2)}(s)\right)>\frac{c}{\lambda} \quad \text { for } t \in[\eta, 1] .
$$

Therefore,

$$
\begin{aligned}
\gamma(T u)= & (T u)^{(n-2)}(\eta) \\
= & -\int_{0}^{\eta}(\eta-s) f\left(s, u(s), u^{\prime}(s), \ldots, u^{(n-2)}(s)\right) d s \\
& +\frac{\eta}{1-\alpha}\left(\int_{0}^{1} f\left(s, u(s), u^{\prime}(s), \ldots, u^{(n-2)}(s)\right) d s\right. \\
& \left.-\alpha \int_{0}^{\eta} f\left(s, u(s), u^{\prime}(s), \ldots, u^{(n-2)}(s)\right) d s\right) \\
= & \int_{0}^{\eta} s f\left(s, u(s), u^{\prime}(s), \ldots, u^{(n-2)}(s)\right) d s \\
& +\frac{\eta}{1-\alpha} \int_{\eta}^{1} f\left(s, u(s), u^{\prime}(s), \ldots, u^{(n-2)}(s)\right) d s \\
\geq & \frac{\eta}{1-\alpha} \int_{\eta}^{1} f\left(s, u(s), u^{\prime}(s), \ldots, u^{(n-2)}(s)\right) d s \\
> & \frac{c}{\lambda}\left(\frac{\eta}{1-\alpha} \int_{\eta}^{1} d s\right) \\
= & \frac{c}{\lambda}\left(\frac{\eta(1-\eta)}{1-\alpha}\right) \\
= & c .
\end{aligned}
$$

Secondly, we show that Lemma 2.4(ii) is fulfilled. We choose $u \in \partial P(\theta, b)$. Then

$$
\theta(u)=\max _{0 \leq t \leq \eta} u^{(n-2)}(t)=u^{(n-2)}(\eta)=b .
$$

This implies

$$
0 \leq u^{(n-2)}(t) \leq b, \quad 0 \leq t \leq \eta, \quad b \leq u^{(n-2)}(t) \leq\left\|u^{(n-2)}\right\|_{\infty}=u^{(n-2)}(1)
$$


for $t \in[\eta, 1]$. Moreover,

$$
\|u\|=\|u\|_{\infty} \leq \frac{1}{\eta} \gamma(u)=\frac{1}{\eta} \theta(u)=b \frac{1}{\eta} .
$$

Thus $u^{(i)}(t) \geq 0$ for all $t \in[0,1], i=0,1, \ldots, n-2$, and

$$
0 \leq u^{(n-2)}(t) \leq b \frac{1}{\eta}, \quad 0 \leq t \leq 1 .
$$

By (B), we have

$$
f\left(s, u(s), u^{\prime}(s), \ldots, u^{(n-2)}(s)\right)<\frac{b}{\xi}, \quad t \in[0,1]
$$

and so

$$
\begin{aligned}
\theta(T u)= & (T u)^{(n-2)}(\eta) \\
= & -\int_{0}^{\eta}(\eta-s) f\left(s, u(s), u^{\prime}(s), \ldots, u^{(n-2)}(s)\right) d s \\
& +\frac{\eta}{1-\alpha}\left(\int_{0}^{1} f\left(s, u(s), u^{\prime}(s), \ldots, u^{(n-2)}(s)\right) d s\right. \\
& \left.\quad-\alpha \int_{0}^{\eta} f\left(s, u(s), u^{\prime}(s), \ldots, u^{(n-2)}(s)\right) d s\right) \\
= & \int_{0}^{\eta} s f\left(s, u(s), u^{\prime}(s), \ldots, u^{(n-2)}(s)\right) d s \\
& +\frac{\eta}{1-\alpha} \int_{\eta}^{1} f\left(s, u(s), u^{\prime}(s), \ldots, u^{(n-2)}(s)\right) d s \\
< & \frac{b}{\xi}\left(\int_{0}^{\eta} s d s+\frac{\eta}{1-\alpha} \int_{\eta}^{1} d s\right) \\
= & \frac{b}{\xi}\left(\frac{1}{2} \eta^{2}+\frac{\eta(1-\eta)}{1-\alpha}\right) \\
= & b .
\end{aligned}
$$

Finally, we verify that Lemma $2.4(\mathrm{iii})$ is also satisfied. It is easy to show that $P(\phi, a) \neq$ $\varnothing$.

Now, let $u \in \partial P(\phi, a)$, then $\phi(u)=\min _{l \leq t \leq 1} u^{(n-2)}(t)=u^{(n-2)}(l)=a$. This means that

$$
a \leq u^{(n-2)}(t) \leq \frac{a}{l}, \quad l \leq t \leq 1
$$

From assumption (C), we have $u^{(i)}(t) \geq 0$ for all $t \in[0,1]$ and $i=0,1, \ldots, n-2$, and

$$
f\left(s, u(s), u^{\prime}(s), \ldots, u^{(n-2)}(s)\right)>\frac{a}{\lambda_{l}} \text { for } t \in[l, 1],
$$


and so

$$
\begin{aligned}
\phi(T u)= & (T u)^{(n-2)}(l) \\
= & -\int_{0}^{l}(l-s) f\left(s, u(s), u^{\prime}(s), \ldots, u^{(n-2)}(s)\right) d s \\
& +\frac{l}{1-\alpha}\left(\int_{0}^{1} f\left(s, u(s), u^{\prime}(s), \ldots, u^{(n-2)}(s)\right) d s\right. \\
= & \int_{0}^{\eta} s f\left(s, u(s), u^{\prime}(s), \ldots, u^{(n-2)}(s)\right) d s \\
& +\int_{\eta}^{l}\left(\frac{l}{1-\alpha}-l+s\right) f\left(s, u(s), u^{\prime}(s), \ldots, u^{(n-2)}(s)\right) d s \\
& +\frac{l}{1-\alpha} \int_{l}^{1} f\left(s, u(s), u^{\prime}(s), \ldots, u^{(n-2)}(s)\right) d s \\
\geq & \frac{l}{1-\alpha} \int_{l}^{1} f\left(s, u(s), u^{\prime}(s), \ldots, u^{(n-2)}(s)\right) d s \\
> & \frac{a}{\lambda_{l}}\left(\frac{l}{1-\alpha} \int_{l}^{1} d s\right) \\
= & \frac{a}{\lambda_{l}}\left(\frac{l(1-l)}{1-\alpha}\right) \\
= & a .
\end{aligned}
$$

Therefore, BVP (1.3)-(1.4) has at least two positive solutions $u_{1}$ and $u_{2}$ in $\overline{P(\gamma, c)}$ such that

$$
a<\phi\left(u_{1}\right) \text { with } \theta\left(u_{1}\right)<b, \quad b<\theta\left(u_{2}\right) \quad \text { with } \gamma\left(u_{2}\right)<c \text {. }
$$

This completes the proof of Theorem 2.8.

Now we deal with the following boundary value problem:

$$
\begin{aligned}
& (-1)^{n} u^{(n)}+f\left(t, u(t), u^{\prime}(t), \ldots, u^{(n-2)}(t)\right)=0, \quad 0<t<1, \\
& u^{(i)}(1)=0, \quad i=0,1, \ldots, n-2, \quad u^{(n-1)}(0)=\alpha u^{(n-1)}(\eta),
\end{aligned}
$$

where $\alpha \geq 0,0<\eta<1$, but fixed, $1-\alpha>0, f:[0,1] \times R^{n-1} \rightarrow R$ is continuous and satisfies

$$
(-1)^{n} f\left(t, u_{0}, u_{1}, \ldots, u_{n-2}\right) \geq 0 \text { for }\left(t, u_{0}, \ldots, u_{n-2}\right) \in[0,1] \times R_{+}^{n-1} .
$$


If $x$ is a solution of BVP (2.41), then

$$
\begin{aligned}
x(t)=-\int_{t}^{1} \frac{(s-t)^{n-1}}{(n-1) !} f\left(s, x(s), x^{\prime}(s), \ldots, x^{(n-2)}(s)\right) d s & \\
+\frac{(1-t)^{n-1}}{(1-\alpha)(n-1) !}[ & \int_{0}^{1} f\left(s, x(s), x^{\prime}(s), \ldots, x^{(n-2)}(s)\right) d s \\
& \left.\quad-\alpha \int_{\eta}^{1} f\left(s, x(s), x^{\prime}(s), \ldots, x^{(n-2)}(s)\right) d s\right] .
\end{aligned}
$$

It is easy to see that

$$
\begin{aligned}
(-1)^{n-2} x^{(n-2)}(t)=- & \int_{t}^{1}(s-t) f\left(s, x(s), x^{\prime}(s), \ldots, x^{(n-2)}(s)\right) d s \\
& +\frac{1-t}{1-\alpha}\left[\int_{0}^{1} f\left(s, x(s), x^{\prime}(s), \ldots, x^{(n-2)}(s)\right) d s\right. \\
& \left.-\alpha \int_{\eta}^{1} f\left(s, x(s), x^{\prime}(s), \ldots, x^{(n-2)}(s)\right) d s\right], \\
(-1)^{n-1} x^{(n-1)}(t)=- & \int_{t}^{1} f\left(s, x(s), x^{\prime}(s), \ldots, x^{(n-2)}(s)\right) d s \\
& +\frac{1}{1-\alpha}\left[\int_{0}^{1} f\left(s, x(s), x^{\prime}(s), \ldots, x^{(n-2)}(s)\right) d s\right. \\
& \left.-\alpha \int_{\eta}^{1} f\left(s, x(s), x^{\prime}(s), \ldots, x^{(n-2)}(s)\right) d s\right], \\
(-1)^{n} x^{(n)}(t)= & -f\left(t, x(t), x^{\prime}(t), \ldots, x^{(n-2)}(t)\right) .
\end{aligned}
$$

Let $E$ denote the Banach space $C^{n-2}[0,1]$ with the norm

$$
\|y\|=\max \left\{\|y\|_{\infty}, \ldots,\left\|y^{(n-2)}\right\|_{\infty}\right\} .
$$

It is easy to see that $\|y\|=\left\|y^{(n-2)}\right\|_{\infty}$ for all $y \in E$. Define the cone $P \subset E$ by

$$
\begin{aligned}
P= & \left\{u \in E: u^{(i)}(1)=0, i=0,1, \ldots, n-2,(-1)^{n-2} u^{(n-2)}(t)\right. \\
& \text { is nondecreasing, } \left.(-1)^{n-2} u^{(n-2)}(t) \geq t\left\|u^{(n-2)}\right\|_{\infty} \text { for } t \in[0,1]\right\} .
\end{aligned}
$$

The method is just similar to what we have done above. We choose a fixed number $l \in$ $(0, \eta)$, and define the nonnegative, increasing functionals $\gamma, \theta$, and $\phi$ on $P$, respectively, as

$$
\begin{aligned}
& \gamma(u)=\min _{l \leq t \leq \eta}(-1)^{n-2} u^{(n-2)}(t)=(-1)^{n-2} u^{(n-2)}(\eta), \\
& \theta(u)=\max _{\eta \leq t \leq 1}(-1)^{n-2} u^{(n-2)}(t)=(-1)^{n-2} u^{(n-2)}(\eta), \\
& \phi(u)=\min _{0 \leq t \leq l}(-1)^{n-2} u^{(n-2)}(t)=(-1)^{n-2} u^{(n-2)}(l) .
\end{aligned}
$$


Define the operator $T: P \rightarrow X$ by

$$
\begin{aligned}
T x(t)=-\int_{t}^{1} \frac{(s-t)^{n-1}}{(n-1) !} f\left(s, x(s), x^{\prime}(s), \ldots, x^{(n-2)}(s)\right) d s \\
+\frac{(1-t)^{n-1}}{(1-\alpha)(n-1) !}\left[\int_{0}^{1} f\left(s, x(s), x^{\prime}(s), \ldots, x^{(n-2)}(s)\right) d s\right. \\
\left.\quad-\alpha \int_{\eta}^{1} f\left(s, x(s), x^{\prime}(s), \ldots, x^{(n-2)}(s)\right) d s\right] .
\end{aligned}
$$

Let

$$
\lambda=\frac{\eta(1-\eta)}{1-\alpha}, \quad \xi=\frac{1}{2}(1-\eta)^{2}+\frac{\eta(1-\eta)}{1-\alpha}, \quad \lambda_{l}=\frac{l(1-l)}{1-\alpha} .
$$

By a method similar to that of Theorem 2.8, we have the following theorem and its proof is omitted.

THEOREM 2.9. Suppose $0<a<\left(\lambda_{r} / \xi\right) b<\eta\left(\lambda_{r} / \xi\right) c$ and $f$ satisfies the following conditions:

(D) $(-1)^{n} f\left(t, w_{0}, w_{1}, \ldots, w_{n-2}\right)>c / \lambda$ for $\left(t, w_{0}, w_{1}, \ldots, w_{n-2}\right) \in[\eta, 1] \times R_{+}^{n-2} \times$ $[c, c / \eta]$

(E) $(-1)^{n} f\left(t, w_{0}, w_{1}, \ldots, w_{n-2}\right)<b / \xi$ for $\left(t, w_{0}, w_{1}, \ldots, w_{n-2}\right) \in[0,1] \times R_{+}^{n-2} \times$ $[0, b / \eta]$

(F) $(-1)^{n} f\left(t, w_{0}, w_{1}, \ldots, w_{n-2}\right)>a / \lambda_{l}$ for $\left(t, w_{0}, w_{1}, \ldots, w_{n-2}\right) \in[l, 1] \times R_{+}^{n-2} \times$ $[a, a / l]$.

Then the BVP (2.41) has at least two positive solutions $u_{1}, u_{2}$ such that

$$
a<\phi\left(u_{1}\right) \quad \text { with } \theta\left(u_{1}\right)<b, \quad b<\theta\left(u_{2}\right) \quad \text { with } \gamma\left(u_{2}\right)<c \text {. }
$$

We now denote $\lambda^{\prime}, \xi^{\prime}$, and $\lambda_{l}^{\prime}$ by

$$
\lambda^{\prime}=\frac{1}{2} \eta^{2}+\frac{\eta(1-\eta)}{1-\alpha}, \quad \xi^{\prime}=\frac{\eta(1-\eta)}{1-\alpha}, \quad \lambda_{l}^{\prime}=\frac{1}{2} l^{2}+\frac{\alpha l(1-\eta)}{1-\alpha}+\frac{l(1-l)}{1-\alpha} .
$$

THEOREM 2.10. Suppose $0<a<l b<(\lambda / \xi) l c$, and $f$ satisfies the following conditions:

( $\left.\mathrm{A}^{\prime}\right) f\left(t, w_{0}, w_{1}, \ldots, w_{n-2}\right)<c / \lambda^{\prime}$ for $\left(t, w_{0}, w_{1}, \ldots, w_{n-2}\right) \in[0,1] \times R_{+}^{n-2} \times[0, c / \eta]$;

(B') $f\left(t, w_{0}, w_{1}, \ldots, w_{n-2}\right)>b / \xi^{\prime}$ for $\left(t, w_{0}, w_{1}, \ldots, w_{n-2}\right) \in[\eta, 1] \times R_{+}^{n-2} \times[b, b / \eta]$;

(C') $f\left(t, w_{0}, w_{1}, \ldots, w_{n-2}\right)<a / \lambda_{l}^{\prime}$ for $\left(t, w_{0}, w_{1}, \ldots, w_{n-2}\right) \in[0,1] \times R_{+}^{n-2} \times[0, a / l]$.

Then the BVP (1.3)-(1.4) admits at least two positive solutions $u_{1}, u_{2}$ such that

$$
a<\phi\left(u_{1}\right) \quad \text { with } \theta\left(u_{1}\right)<b, \quad b<\theta\left(u_{2}\right) \quad \text { with } \gamma\left(u_{2}\right)<c \text {. }
$$


We denote $\lambda^{\prime}, \xi^{\prime}$, and $\lambda_{r}^{\prime}$ by

$$
\begin{gathered}
\lambda^{\prime}=\frac{1}{2}(1-\eta)^{2}+\frac{\eta(1-\eta)}{1-\alpha}, \\
\xi^{\prime}=\frac{\eta(1-\eta)}{1-\alpha}, \\
\lambda_{r}^{\prime}=\frac{1}{2} l^{2}+\frac{l(1-l)}{1-\alpha}+\left(\frac{1-l}{1-\alpha}+\eta\right)(\eta-l)+(1+\eta-l)(1-\eta)-\frac{1}{2} .
\end{gathered}
$$

THEOREM 2.11. Suppose $0<a<l b<\left(\xi^{\prime} / \lambda^{\prime}\right) l c$, and $f$ satisfies the following conditions:

(D') $(-1)^{n} f\left(t, w_{0}, w_{1}, \ldots, w_{n-2}\right)<c / \lambda^{\prime}$ for $\left(t, w_{0}, w_{1}, \ldots, w_{n-2}\right) \in[0,1] \times R_{+}^{n-2} \times$ $[0, c / \eta]$;

(E') $(-1)^{n} f\left(t, w_{0}, w_{1}, \ldots, w_{n-2}\right)>b / \xi^{\prime}$ for $\left(t, w_{0}, w_{1}, \ldots, w_{n-2}\right) \in[0, \eta] \times R_{+}^{n-2} \times$ $[b, b / \eta]$

$\left(\mathrm{F}^{\prime}\right)(-1)^{n} f\left(t, w_{0}, w_{1}, \ldots, w_{n-2}\right)<a / \lambda_{l}^{\prime}$ for $\left(t, w_{0}, w_{1}, \ldots, w_{n-2}\right) \in[0,1] \times R_{+}^{n-2} \times$ $[0, a / l]$.

Then the BVP (2.41) admits at least two positive solutions $u_{1}, u_{2}$ such that

$$
a<\phi\left(u_{1}\right) \quad \text { with } \theta\left(u_{1}\right)<b, \quad b<\theta\left(u_{2}\right) \quad \text { with } \gamma\left(u_{2}\right)<c \text {. }
$$

3. Applications. In this section, we present the theorems which may be considered as the corollaries of Theorems 2.8, 2.9, 2.10, and 2.11, respectively.

THEOREM 3.1. Suppose that

(i) $f_{0}=\lim _{x_{n-2} \rightarrow 0}\left(f\left(t, x_{0}, \ldots, x_{n-2}\right) / x_{n-2}\right)=+\infty, f_{\infty}=\lim _{x_{n-2} \rightarrow+\infty}\left(f\left(t, x_{0}, \ldots\right.\right.$, $\left.x_{n-2}\right)\left(x_{n-2}\right)=+\infty$ are uniform in $t, x_{0}, \ldots, x_{n-2}$;

(ii) there is $0<\eta<l<1$ and $x_{0}>0$ such that

$$
f\left(t, x_{0}, \ldots, x_{n-2}\right)<\frac{\eta}{\xi} x_{0} \quad \text { for }\left(t, x_{0}, \ldots, x_{n-2}\right) \in[0,1] \times R_{+}^{n-2} \times\left[0, x_{0}\right],
$$

where $\lambda, \xi$, and $\lambda_{l}$ are given in Theorem 2.8 .

Then BVP (1.3)-(1.4) has at least two positive solutions.

Proof. Firstly, by (ii), choosing $b=x_{0} \eta$, one gets

$$
f\left(t, w_{0}, \ldots, w_{n-2}\right)<\frac{1}{\xi} b \quad \text { for } 0 \leq w_{n-2} \leq x_{0},\left(t, w_{0}, \ldots, w_{n-3}\right) \in[0,1] \times R_{+}^{n-3} .
$$

Secondly, choose $K$ sufficiently large such that

$$
K \lambda=K\left(\frac{\eta(1-\eta)}{1-\alpha}\right)>1
$$

Since $f_{0}=+\infty$, there is $R_{1}>0$ sufficiently small such that

$$
f\left(t, x_{0}, \ldots, x_{n-2}\right) \geq K x_{n-2} \text { for } 0 \leq x_{n-2} \leq R_{1},\left(t, x_{0}, \ldots, x_{n-3}\right) \in[0,1] \times R_{+}^{n-3} \text {. }
$$


Without loss of generality, suppose $R_{1} \leq\left(\lambda_{r} / \xi\right)(1 / \eta) b$. Choose $a>0$ so that $a<l R_{1}$ and $a<\left(\lambda_{r} / l\right) b$. For $a \leq w_{n-2} \leq(1 / l) a$, we have $w_{n-2} \leq R_{1}$. Thus

$$
\begin{aligned}
& f\left(t, w_{0}, \ldots, w_{n-2}\right) \geq K w_{n-2} \geq K a>\frac{a}{\lambda} \\
& \qquad \text { for } a \leq w_{n-2} \leq \frac{1}{\eta} a,\left(t, x_{0}, \ldots, x_{n-3}\right) \in[0,1] \times R_{+}^{n-2} .
\end{aligned}
$$

Thirdly, choose $K_{1}$ sufficiently large such that

$$
K_{1} \xi=K_{1}\left(\frac{l(1-l)}{1-\alpha}\right)>1
$$

Since $f_{\infty}=\infty$, there is $R_{2}>0$ sufficiently large such that

$$
f\left(t, x_{0}, \ldots, x_{n-2}\right) \geq K_{1} x_{n-2} \text { for } x_{n-2} \geq R_{2},\left(t, x_{0}, \ldots, x_{n-3}\right) \in[0,1] \times R_{+}^{n-3} .
$$

Without loss of generality, suppose $R_{2}>(1 / \eta) b$. Choose $c \geq R_{2}$. Then

$$
\begin{aligned}
& f\left(t, w_{0}, \ldots, w_{n-2}\right) \geq K_{1} w_{n-2} \geq K_{1} c>\frac{c}{\lambda_{l}} \\
& \quad \text { for } c \leq w_{n-2} \leq \frac{1}{l} c,\left(t, x_{0}, \ldots, x_{n-3}\right) \in[0,1] \times R_{+}^{n-3} .
\end{aligned}
$$

Hence, it follows from the definition of $a, b$, and $c$ that

$$
0<a<\frac{\lambda_{l}}{\xi} b<\eta \frac{\lambda_{l}}{\xi} c
$$

and conditions in Theorem 2.8 are satisfied. By Theorem 2.8, BVP (1.3)-(1.4) has at least two positive solutions. The proof is complete.

THEOREM 3.2. Suppose that

(i) $f_{0}=\lim _{x_{n-2} \rightarrow 0}\left(f\left(t, x_{0}, \ldots, x_{n-2}\right) / x_{n-2}\right)=\infty, f_{\infty}=\lim _{x_{n-2} \rightarrow+\infty}\left(f\left(t, x_{0}, \ldots, x_{n-2}\right) /\right.$ $\left.x_{n-2}\right)=\infty$ are uniform in $t, x_{0}, \ldots, x_{n-2}$;

(ii) there are $0<l<\eta<1$ and $x_{0}>0$ such that

$$
\frac{f\left(t, x_{0}, \ldots, x_{n-2}\right)}{1-\eta}<\frac{\eta}{\xi} x_{0} \quad \text { for }\left(t, x_{0}, \ldots, x_{n-2}\right) \in[0,1] \times R_{+}^{n-3} \times\left[0, x_{0}\right]
$$

where $\lambda, \xi$, and $\lambda_{l}$ are given in Theorem 2.9 .

Then BVP (2.41) has at least two positive solutions. 
Proof. The proof is similar to that of Theorem 3.1 and is omitted.

THEOREM 3.3. Suppose that

(i) $f_{0}=\lim _{x_{n-2} \rightarrow 0}\left(f\left(t, x_{0}, \ldots, x_{n-2}\right) / x_{n-2}\right)=0, f_{\infty}=\lim _{x_{n-2} \rightarrow+\infty}\left(f\left(t, x_{0}, \ldots\right.\right.$, $\left.x_{n-2}\right)\left(x_{n-2}\right)=0$ are uniform in $t, x_{0}, \ldots, x_{n-2}$;

(ii) there are $0<\eta<l<1$ and $x_{0}>0$ such that

$f\left(t, x_{0}, \ldots, x_{n-2}\right)>\frac{\eta}{\xi^{\prime}} x_{0} \quad$ for $\left(t, x_{0}, \ldots, x_{n-2}\right) \in[\eta, 1] \times R_{+}^{n-3} \times\left[0, x_{0}\right]$,

where $\xi^{\prime}$ is given in Theorem 2.10.

Then BVP (1.3)-(1.4) has at least two positive solutions.

THEOREM 3.4. Suppose that

(i) $f_{0}=\lim _{x_{n-2} \rightarrow 0}\left(f\left(t, x_{0}, \ldots, x_{n-2}\right) / x_{n-2}\right)=0, f_{\infty}=\lim _{x_{n-2} \rightarrow+\infty}\left(f\left(t, x_{0}, \ldots\right.\right.$, $\left.x_{n-2}\right)\left(x_{n-2}\right)=0$ are uniform in $t, x_{0}, \ldots, x_{n-2}$;

(ii) there are $0<l<\eta<1$ and $x_{0}>0$ such that

$$
\frac{f\left(t, x_{0}, \ldots, x_{n-2}\right)}{1-\eta}>\frac{\eta}{\xi^{\prime}} x_{0} \quad \text { for }\left(t, x_{0}, \ldots, x_{n-2}\right) \in[0, \eta] \times R_{+}^{n-3} \times\left[0, x_{0}\right],
$$

where $\xi^{\prime}$ is given in Theorem 2.11 .

Then BVP (2.41) has at least two positive solutions.

Proof. The proofs of Theorems 3.3 and 3.4 are similar to that of Theorem 3.1 and are omitted.

ACKNOWLEDGMENT. The first author is supported by the Science Foundation of Educational Committee of Hunan Province and both authors are supported by the National Natural Science Foundation of China.

\section{REFERENCES}

[1] R. P. Agarwal, Boundary Value Problems for Higher Order Differential Equations, World Scientific Publishing, New Jersey, 1986.

[2] R. P. Agarwal and D. O'Regan, Singular differential, integral and discrete equations: the semipositone case, Mosc. Math. J. 2 (2002), no. 1, 1-15.

[3] R. P. Agarwal, D. O'Regan, and P. J. Y. Wong, Positive Solutions of Differential, Difference and Integral Equations, Kluwer Academic Publishers, Dordrecht, 1999.

[4] D. R. Anderson, Multiple positive solutions for a three-point boundary value problem, Math. Comput. Modelling 27 (1998), no. 6, 49-57.

[5] D. R. Anderson and J. M. Davis, Multiple solutions and eigenvalues for third-order right focal boundary value problems, J. Math. Anal. Appl. 267 (2002), no. 1, 135-157.

[6] R. I. Avery, C. J. Chyan, and J. Henderson, Twin solutions of boundary value problems for ordinary differential equations and finite difference equations, Comput. Math. Appl. 42 (2001), no. 3-5, 695-704.

[7] W. Feng, On an M-point boundary value problem, Nonlinear Anal. 30 (1997), no. 8, 53695374.

[8] W. Feng and J. R. L. Webb, Solvability of m-point boundary value problems with nonlinear growth, J. Math. Anal. Appl. 212 (1997), no. 2, 467-480.

[9] D. Guo and V. Lakshmikantham, Nonlinear Problems in Abstract Cones, Notes and Reports in Mathematics in Science and Engineering, vol. 5, Academic Press, Massachusetts, 1988. 
[10] C. P. Gupta, Solvability of a three-point nonlinear boundary value problem for a second order ordinary differential equation, J. Math. Anal. Appl. 168 (1992), no. 2, 540-551.

[11] _ A generalized multi-point boundary value problem for second order ordinary differential equations, Appl. Math. Comput. 89 (1998), no. 1-3, 133-146.

[12] C. P. Gupta and S. I. Trofimchuk, A sharper condition for the solvability of a three-point second order boundary value problem, J. Math. Anal. Appl. 205 (1997), no. 2, 586597.

[13] V. A. Il'in and E. I. Moiseev, Nonlocal boundary value problem of the first kind for a Sturm-Liouville operator in its differential and finite difference aspects, Differ. Equ. 23 (1987), no. 7, 803-810.

[14] B. Liu, Positive solutions of a nonlinear three-point boundary value problem, Comput. Math. Appl. 44 (2002), no. 1-2, 201-211.

[15] R. Ma, Positive solutions for second-order three-point boundary value problems, Appl. Math. Lett. 14 (2001), no. 1, 1-5.

Yuji Liu: Department of Applied Mathematics, Beijing Institute of Technology, Beijing 100081, China

Current address: Department of Mathematics, Hunan Institute of Technology, Yueyang, Hunan 414000, China

E-mail address: 7 iuyuji888@sohu.com

Weigao Ge: Department of Applied Mathematics, Beijing Institute of Technology, Beijing 100081, China

E-mail address: gew@ibit.edu.cn 


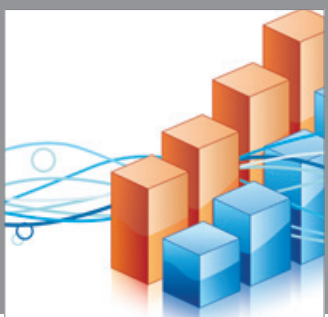

Advances in

Operations Research

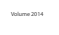

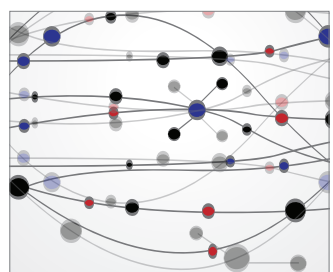

\section{The Scientific} World Journal
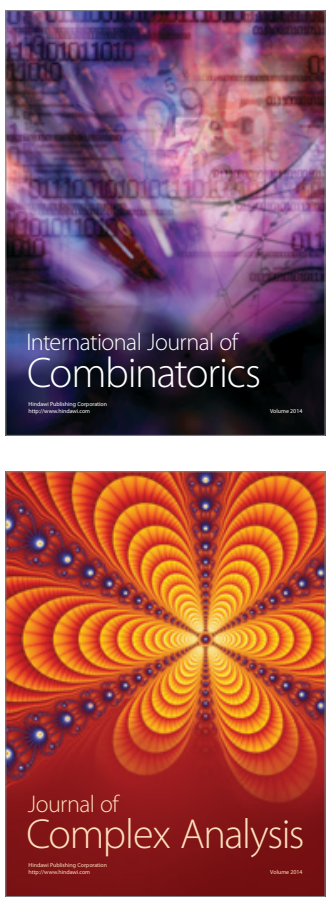

International Journal of

Mathematics and

Mathematical

Sciences
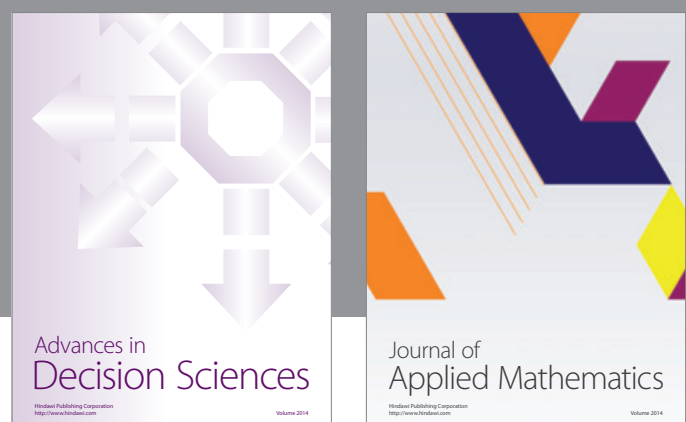

Journal of

Applied Mathematics
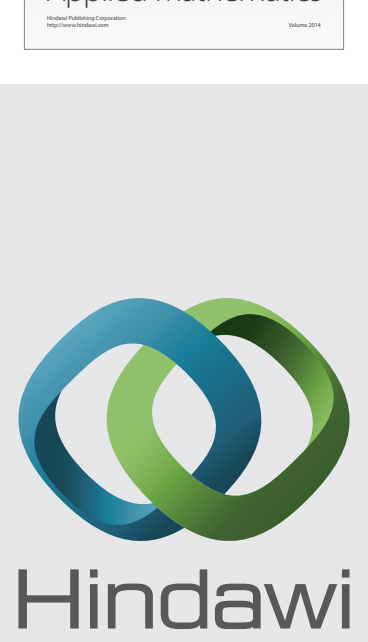

Submit your manuscripts at http://www.hindawi.com
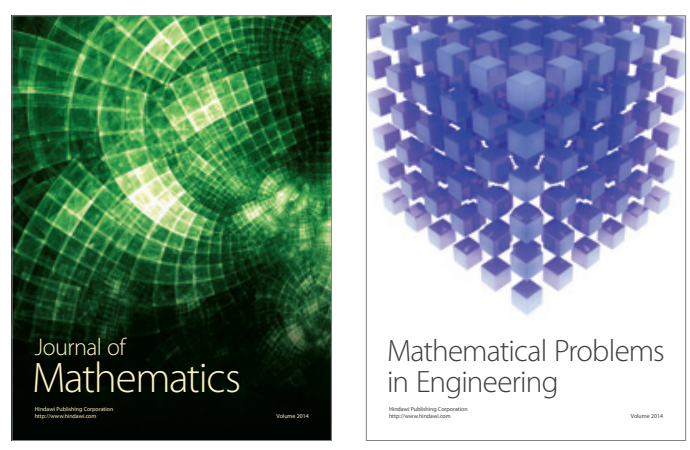

Mathematical Problems in Engineering
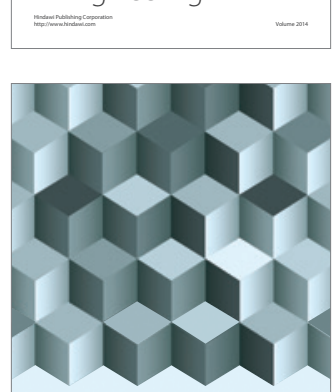

Journal of

Function Spaces
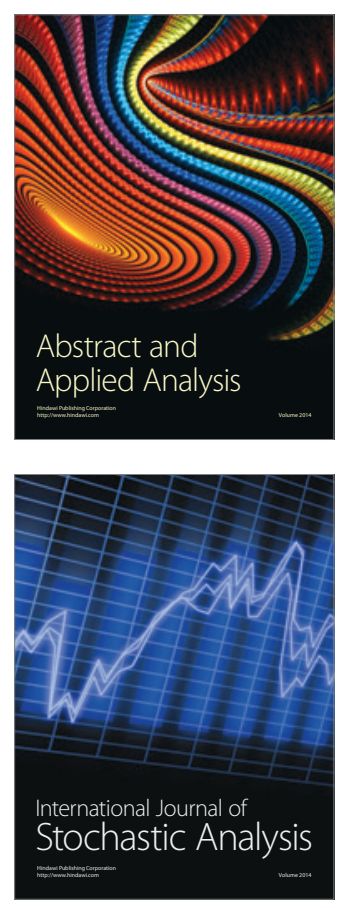

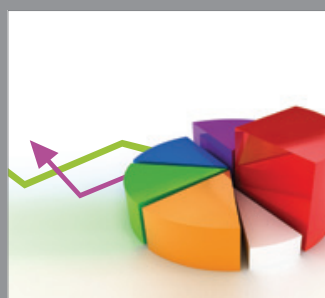

ournal of

Probability and Statistics

Promensencen
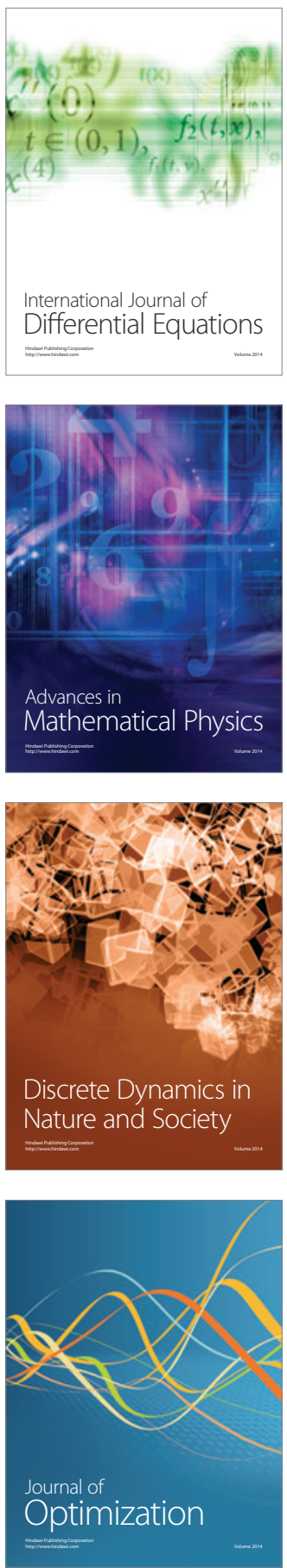